\title{
Sobre Problemas en torno a la desigualdad. Un enfoque poliédrico, de Esteban Llamosas y Guillermo Lariguet (eds.)
}

\author{
On Problemas en torno a la desigualdad. Un \\ enfoque poliédrico, by Esteban Llamosas and \\ Guillermo Lariguet (eds.)
}

Ilsse Carolina Torres Ortega ${ }^{*}$

Recepción: 03/07/2021

Evaluación: 30/07/2021

Aceptación final: 18/08/2021

\begin{abstract}
Resumen: En esta reseña se presentan y articulan algunas de las aportaciones contenidas en el libro Problemas en torno a la desigualdad. Un enfoque poliédrico. Esta obra, coordinada por Esteban Llamosas y Guillermo Lariguet, reúne una serie de trabajos elaborados por profesoras y profesores de la Universidad Nacional de Córdoba que, desde el ámbito de la sociología, de la psicología y la filosofía política, y de la historia y la sociología del Derecho, reflexionan sobre los retos de las desigualdades y la posibilidad de acción sobre ellas.
\end{abstract}

Palabras clave: desigualdades, igualdad, agencia, percepción, transformación social.

\footnotetext{
Doctora en Derecho por la Universidad de Alicante. Profesora investigadora en el Departamento de Estudios Sociopolíticos y Jurídicos del Instituto Tecnológico de Estudios Superiores de Occidente (ITESO) (Jalisco, México) y miembro del Sistema Nacional de Investigadores de México. Código ORCID: 0000-0002-5929-9137. Correo electrónico: torresilsse@ iteso.mx
} 


\begin{abstract}
This review presents and articulates some of the contributions contained in the book Problemas en torno a la desigualdad. Un enfoque poliédrico. This work, coordinated by Esteban Llamosas and Guillermo Lariguet, brings together a series of papers written by professors from the National University of Córdoba who, from the fields of sociology, psychology and political philosophy, and the history and sociology of law, reflect on the challenges of inequalities and the possibility of action on them.
\end{abstract}

Keywords: inequalities, equality, agency, perception, social transformation.

\title{
1. Introducción
}

A día de hoy resultan cada vez más habituales en el discurso público ideas como las expresadas por Milton y Rose Friedman en Free to Choose: "La vida no es justa. Es tentador creer que el gobierno puede rectificar lo que la naturaleza ha engendrado. Pero también es importante reconocer lo mucho que nos beneficiamos de la misma injusticia que deploramos" (1980, p. 137). Para muchos lectores, palabras como las anteriores pueden resultar burdas, incluso escandalosas; sin embargo, reflejan una actitud bastante extendida socialmente que pretende justificar la injusticia, naturalizándola y presentándola como éticamente neutral. Estamos tan habituados a las injusticias que estas han pasado a formar parte del paisaje moral del mundo y de la vida en común.

La afirmación y la aceptación de que la vida es injusta y de que siempre lo será nos lleva directo a los derroteros de las desigualdades. Ya Aristóteles establecía que lo injusto es desigual (1998, p. 249). Las desigualdades están tan enraizadas en nuestra historia y en el día a día de nuestras acciones más cotidianas que resulta sencillo no solo habituarse a ellas, sino buscar explicaciones y justificaciones que terminan por perpetuarlas, incorporándolas al entendimiento de lo que es y debe ser la humanidad. Así, al igual que con las palabras de Friedman, se alienta un discurso del cinismo respecto a que las personas y sus circunstancias de vida son inevitablemente desiguales, quedando en las manos de cada uno beneficiarse o perjudicarse de ello.

El libro Problemas en torno a la desigualdad. Un enfoque poliédrico coordinado por Esteban Llamosas y Guillermo Lariguet (2020) es un 
esfuerzo por refutar todo lo anterior. Las personas, las cosas, las circunstancias están atravesadas por profundas desigualdades - entendidas en el sentido descriptivo de propiedades ontológicas-, pero hay algunas de ellas que dan lugar a estados de cosas y tratamientos que resultan intolerables en comunidades que erigen un compromiso con una ética de mínimos. ${ }^{1}$ Se trata de esas desigualdades que - siguiendo el título del texto de Cristián Fatauros, incluido en el libro "Las desigualdades que nos duelen, ¿son acaso desigualdades que deberíamos tolerar?” (pp. 93-106) - "nos duelen” porque detrás de ellas hay seres humanos que padecen, oportunidades que se ven coartadas y planes de vida que ni siquiera llegan a concebirse. Hay desigualdades que configuran el mundo en que vivimos, pero también el mundo que percibimos.

Esta obra coordinada agrupa una serie de trabajos elaborados por profesores de la Universidad de Córdoba, Argentina, en un diálogo académico a propósito de la desigualdad y el Derecho. Estas profesoras y profesores se acercan desde distintas perspectivas, formaciones e intereses a un fenómeno que, aunque extremadamente familiar, parece tener poca relevancia en la deliberación pública, siendo necesario introducirlo en esta cuanto antes. Sin embargo, tal y como indica Hugo Seleme en su capítulo, "El lugar de la democracia y la magnitud de la desigualdad" (pp. 79-92), para que un problema social se convierta en un problema público es necesario que sea percibido adecuadamente por la ciudadanía. De otra forma, "el mal se vuelve invisible, la invisibilidad lo hace intangible y la intangibilidad, finalmente, lo transforma en invencible" (2020, p. 79).

Las desigualdades conforman un fenómeno tan complejo que lleva a las autoras y autores del libro a revisar su instrumental disciplinario y a reconocer que se requiere de muchas más herramientas teórico-prácticas para dar cuenta de las desigualdades y para intentar paliarlas. Me atrevería a decir que en esto último recae el rasgo característico de la obra. A este esfuerzo por comunicar y desdibujar las barreras del conocimiento, sub-

1 Aquí hago referencia a la distinción planteada por A. Cortina y E. Martínez entre ética de máximos (que se refiere a lo bueno, a las éticas de la felicidad que intentan ofrecer ideales de vida buena) y la de mínimos (referida a lo justo, a aquellos deberes de justicia que pueden ser exigibles a cualquier ser racional) (2001, p. 117). 
yace una aserción en común: que el trabajo investigativo - la reflexión, el diálogo y la acción con otros - tiene el potencial de cambiar el estado de las cosas. No se trata, pues, de un ejercicio de erudición frívolo, sino de una acción del académico comprometido que aspira a la transformación social. ${ }^{2}$

El libro se divide en dos grandes apartados. El primero denominado "Hacia una teoría de la(s) desigualdad(es)" pretende aportar elementos de reflexión sobre "aquello que puede conceptual, empírica, histórica o normativamente contar como una desigualdad" (Llamosas y Lariguet, 2020, p. 10). La segunda parte, bajo el título "Dimensiones aplicadas al problema de la desigualdad" recoge algunos ejemplos de teoría aplicada, subrayando las dificultades y debilidades que median en esta aplicación. Debido a que son innumerables las aportaciones contenidas en esta obra y a que sería poco sistemático intentar dar cuenta de todas ellas, a continuación, presento una serie de tópicos que, me parece, recogen algunas de las principales preocupaciones en común de estos diversos aportes. El objetivo de agrupar las ideas de esta forma es intentar hacer justicia al libro, mostrando el calado de las problemáticas que aborda. También y especialmente invitar al lector de esta reseña a acercarse a la obra y a participar en la construcción del conocimiento sobre las desigualdades.

\section{Desigualdades y ausencia de igualdad}

Una de las cuestiones que se ponen de manifiesto desde las primeras páginas de la obra es la necesidad de plantear la desigualdad - las desigualdades - como una problemática que requiere de cierta autonomía en su estudio. Por así decirlo, es preciso tematizar las desigualdades. El enfoque de las desigualdades entendidas solo como la ausencia de igualdad no logra

En esto sigo la tipología de intelectuales propuesta por Guillermo Lariguet (2018). El intelectual comprometido no es aquel que milita en un partido o forma parte del gobierno en turno, sino aquel que posee un compromiso cívico resultado de conjugar virtudes intelectuales (poder reflexivo fuerte, no ingenuidad, falta de ceguera o sordera a las buenas razones de los otros) con virtudes morales (como la humildad, la valentía, la integridad) que le llevan a mantener una participación activa en la vida democrática, pero desde una posición crítica (p. 198). 
dar cuenta de la magnitud del fenómeno y, además, metodológicamente dificulta su abordaje. Tal y como indica Lariguet en "Prólogo. La desigualdad: una triste e infinita melodía” (2020, pp. 13-29), a diferencia del término igualdad, que suele ir acompañado de calificativos finitos, resultado de la intensa reflexión que ha permitido distinguir y acotar sus diversas dimensiones de análisis, en el caso de la palabra desigualdad dichos calificativos parecen ser infinitos: "así como el espectro de los dolores del alma es amplísimo, variado, profuso, el rango de cuentas pendientes en materia de igualdad es tan bochornosamente grande, que las predicaciones de lo desigual son casi infinitas" (p. 15). Con el ánimo de avanzar en la concreción de lo infinito de las desigualdades, en el libro se presentan reflexiones desde el ámbito de la sociología, de la psicología y la filosofía política, y de la historia y la sociología del Derecho, refiriendo con ello un objeto de estudio diferenciado, susceptible de ser estudiado desde distintas perspectivas.

Sin embargo, esto no quiere decir que la reflexión sobre el valor de la igualdad como tal se encuentre ausente. El trabajo de Esteban Llamosas, "Las desigualdades jurídicas: de naturales a invisibles, entre el Antiguo Régimen y la codificación” (2020, pp. 65-78), por ejemplo, pone de manifiesto el contraste entre dos maneras distintas de concebir la igualdad. Una en la que la igualdad no es el referente inmediato de la justicia, sino el criterio que permite reconocer y establecer ciertas diferencias naturales entre los seres humanos que asignan estatus distintos a los miembros de un grupo social. La otra concepción ve en la igualdad un presupuesto de los ideales liberales, aunque sea solo una igualdad normativa, declarada en los textos fundantes del orden social. Una aproximación semejante la encontramos en el texto de Cristián Fatauros, quien destaca el papel fundamental del valor de la igualdad en el ámbito jurídico, ya que se trata de un presupuesto de la justicia y de la posibilidad de la democracia. A partir de la igualdad se establece la prerrogativa elemental de moralidad pública de tratar a la ciudadanía con igual respeto y consideración. Esta igualdad de trato y de derechos es la base de la legitimidad de cualquier gobierno, aunque esto contraste con el desprecio y la sospecha que suscita hablar de igualdad en un ambiente asediado por la concepción del mérito y de la responsabilidad individual como criterios de distribución de bienes y ventajas sociales. Y es así que: "cualquier atisbo de mención de políticas sociales que tengan 
como objetivo promover la igualdad de las personas automáticamente es atacada y denostada por 'populista' o 'socialista”' (Fatauros, 2020, p. 94).

El trabajo de Marisa Fassi y Angélica Peñas, "Colonialidad y pandemia 2.0. Retos para repensar los relatos sobre el trabajo sexual en Argentina" (2020, pp. 109-121), con su invitación a revisar y desconfiar de los binomios, resulta sugerente para revisar cómo la dicotomía igualdad/ausencia de igualdad tiene efectos totalizadores en cada categoría que vienen a desdibujar las complejidades y las relaciones de poder que hay entre ellas. Las desigualdades no pueden entenderse solo como la ausencia de igualdad porque hay múltiples ocasiones en las que el camino hacia la justicia es el reconocimiento de la diferencia y su dignificación. La urgencia por reconducir esas desigualdades hacia la igualdad lleva con frecuencia a ejercicios de simulación que terminan por oprimir e invisibilizar a determinados colectivos. En definitiva, dedicar todos los esfuerzos intelectuales hacia la comprensión del valor de la igualdad, pensando que con ello se abarca el vasto terreno de las desigualdades es reduccionista e impide analizar sus causas y consecuencias, también a proponer acciones para mitigarlas. Hay desigualdades que no podemos eliminar, pero sobre las cuales podemos intervenir para prevenirlas o contener sus efectos. Esto, sin embargo, exige un conocimiento y una sensibilidad mayor en torno a los mecanismos que las configuran y las perpetúan.

\section{Agencia moral vs. mero azar}

Una vez iniciado el estudio diferenciado de las desigualdades, una cuestión fundamental a determinar es la distinción de tipos de desigualdades. Como se mencionó anteriormente, sin embargo, hay desigualdades que tienen que ver con la diferencia de características entre los seres y las cosas. Hay características que se comparten y otras que no. Es este un sentido puramente descriptivo de igualdad y desigualdad. Pero hay otras desigualdades que trascienden esa dimensión descriptiva y llegan hasta lo normativo. Esas desigualdades apelan entonces a un trato diferenciado; i. e. establecen una diferencia normativa entre los seres humanos. 
Algunas de estas desigualdades pueden considerarse justificadas si, por ejemplo, mejoran la situación de los menos favorecidos, como lo propone el segundo principio de justicia de John Rawls (1999). Sin embargo, hay desigualdades que establecen una forma de estar en el mundo en la que unos tienen beneficios y privilegios extendidos, a cambio de que otros resientan las cargas y las dificultades. Son estas últimas las desigualdades que no parecen estar justificadas y que difícilmente puede entenderse que sean toleradas en nuestras comunidades políticas. No obstante, a cualquier intento de tipología de las desigualdades subyace una reflexión más amplia respecto al criterio para discriminar cuándo una desigualdad está o no justificada.

Uno de los criterios más socorridos para hacer esta evaluación consiste en el mérito y la responsabilidad individual. De acuerdo con este criterio, aunque es cierto que las circunstancias de vida de las personas son dispares y que algunas son mejores que otras, llega un momento en que nuestra fortuna o desgracia solo es atribuible a nosotros mismos. Sin embargo, "lo que es merecido" puede entenderse de diversas formas. Por ejemplo, Michael Sandel ha popularizado los peligros de la "tiranía del mérito", la soberbia meritocrática que legitima una política de la humillación ejercida por los "ganadores" sobre los "perdedores": "La idea de que nuestro destino está en nuestras manos, de que 'puedes conseguirlo si pones empeño en ello', es una espada de doble filo: inspiradora por uno de sus bordes, pero odiosa por el otro. Congratula a los ganadores, pero denigra a los perdedores y afecta incluso a la percepción que estos tienen de sí mismos" (Sandel, 2020, p. 14). Hay también algunas versiones más edificantes. Lariguet, por ejemplo, nos recuerda cómo lo merecido desde la teoría clásica de la justicia aristotélica tiene que ver con lo justo y que, en ese sentido, merecer se acerca a la idea de agencia, a la intuición básica de que somos actores de nuestro propio destino (Lariguet, 2020, p. 22).

La noción de agencia moral es tan relevante en nuestra forma de entender la vida - en sus dimensiones autorreferente e intersubjetiva- que resulta controversial concederle al azar un papel destacado en nuestras trayectorias vitales. Por una parte, aceptar que nuestra buena o mala fortuna está fuera de nuestro control implica aceptar una forma de indeterminismo filosófico - todo es azaroso-y, al tiempo, negar la posibilidad del libre 
albedrío. Sin embargo, ignorar el papel que juega el azar y su arbitrariedad moral invisibiliza desigualdades estructurales que repercuten especialmente en los menos afortunados. La neutralización del peso de la suerte probablemente tiene mucho que ver con aquello que Gonzalo Assusa, en "El ser social y la consciencia. Preguntas sobre la dimensión simbólica de la desigualdad social” (2020, pp. 33-49), denuncia en su trabajo: la posición y la autopercepción en la estructura social no suelen coincidir. Hay un porcentaje muy alto de la población que se considera clase media, una tendencia a rechazar la autopercepción de pobreza y una negación por parte de las élites a declararse como la clase privilegiada. Esta distorsión se vincula con los estigmas que acompañan a las categorías: ya sea por la pesada carga de aceptar que uno merece su posición menos privilegiada o por la irritación que genera ver cuestionado el mérito sobre su mejor posición.

Cristián Fatauros también aborda esta cuestión. Los discursos igualitarios despiertan recelo porque, en principio, parecen desincentivar el esfuerzo y la ambición en el proyecto vital. Además, en esta línea, las políticas igualitarias parecen una especie de reivindicación de la envidia que sienten los menos afortunados ante el éxito de otros. Fatauros nos recuerda el argumento rawlsiano de que el azar natural y las contingencias sociales son factores moralmente arbitrarios y que, por tanto, no pueden constituir criterios de distribución de los bienes sociales. Incluso la disposición al esfuerzo parece estar influida por múltiples factores, por lo que no es posible afirmar de manera concluyente que nuestra buena o mala fortuna depende del esfuerzo que pongamos en ello. Sin embargo, esta posición bien puede ser matizada a partir de la de otros autores para quienes algunas desigualdades no son resultado del mero azar, sino de decisiones voluntarias, por lo que, atendiendo a la dignidad humana, deben ser respetadas. Esta última posición subraya sin duda un rasgo esencial de la noción de agencia; ahora bien, esto no quiere decir que reconocer el peso de los factores que intervienen en las vidas de las personas derive en la irresponsabilidad: "El instrumental teórico que apela a la dicotomía responsabilidad/irresponsabilidad, o elecciones/circunstancias, es poco adecuado para lidiar con problemas estructurales, y es inapropiado para evaluar la situación de los que están en la peor posición” (Fatauros, 2020, p. 100). 
Las desigualdades no son relevantes solo en un momento específico de los proyectos vitales; las hay tan arraigadas que requieren de la intervención estatal para distribuir los bienes sociales de tal forma que mitiguen su impacto negativo en los más desfavorecidos. Con independencia de que los seres humanos podamos vernos recompensados por nuestro esfuerzo y ambición, hay desigualdades que parecen escapar de la individualidad; desigualdades que trascienden las vidas aisladas y requieren una comprensión más integral sobre las estructuras de poder que las soportan, las facilitan y las eternizan.

\section{La percepción y la autopercepción de las desigualdades}

Antes he hablado de la búsqueda de un criterio que permita discernir qué desigualdades pueden considerarse justificadas y cuáles no. Un criterio bastante socorrido, decía, es el del mérito y la responsabilidad individual. Otro argumento también explorado es el de las necesidades básicas.

Aunque, en principio, todos estaríamos de acuerdo en que es posible constatar empíricamente ciertas necesidades humanas, no todos los teóricos están de acuerdo con que sea relevante dar a algunas necesidades el estatus de "básicas". ${ }^{3}$ Los enfoques que parten de una visión teleológica de la naturaleza humana suelen tener por asumido ese reconocimiento, aunque muchas veces se ha puesto en duda que esto sea necesario, optando, más bien, por dar importancia a los deseos y preferencias (Nino, 1990, p. 21). No obstante los desacuerdos, es posible afirmar que hay ciertas necesidades cuyo reconocimiento y satisfacción son fundamentales para dotar

3 Sobre el concepto de necesidades básicas hay muchas controversias. Un autor cuyos trabajos son clave en relación con este tema es David Wiggins, quien, para aportar claridad, distingue entre un sentido de necesidad instrumental y uno categórico o absoluto. Cuando la satisfacción de una necesidad es un fin en sí mismo (este fin estaría relacionado con la evitación de un daño), dicha necesidad es absoluta; mientras que la necesidad se considera instrumental si es un medio para alcanzar otro fin. La conexión entre estos dos sentidos de necesidad puede establecerse en dos equivalencias: 1. Yo necesito (categóricamente) X, si y solo si, necesito (instrumentalmente) X para evitar un daño. 2. Yo necesito (instrumentalmente) X para evitar un daño, si y solo si, es necesario, siendo las cosas como realmente son, que para evitar un daño entonces tenga X (Wiggins y Dermen, 1987, p. 64). 
de contenido a la autonomía personal, independientemente de los deseos o preferencias de los agentes. Reconocer ciertas necesidades como básicas es aceptar que tienen fuerza normativa y que entonces es legítimo exigir que sean satisfechas y condenar su insatisfacción como un daño al ser humano. ${ }^{4}$

Reconocer que hay ciertos bienes que satisfacen necesidades que, a su vez, posibilitan la autonomía permite visibilizar una dimensión objetiva de las desigualdades. Las desigualdades intolerables serían aquellas que guarnecen necesidades básicas insatisfechas. Sin embargo, esto no resuelve el problema. La posibilidad de observar desde un punto de vista imparcial y objetivo estas carencias se enfrenta a múltiples dificultades, algunas que tienen que ver con la percepción general de las desigualdades, pero también con la autopercepción de los actores dentro de ellas.

Gonzalo Assusa indica cómo desde la perspectiva sociológica se ha priorizado la determinación de la posición de cada individuo en la estructura social, atendiendo a factores objetivos como el ingreso o el nivel educativo cuando hay un estatus social subjetivo - la autoimagen de clase- que obstruye la reconstrucción de esta estructura. La autopercepción colapsa con las categorías de clase, presentando un problema práctico y epistemológico importante, “¿cuánto de la desigualdad social es lo que se percibe?, ¿cuánto de la realidad del funcionamiento de la desigualdad estriba en su representación?" (Assusa, 2020, p. 42). En definitiva, es sumamente complejo tener una representación fiel de las desigualdades cuando las personas no se identifican con las categorías utilizadas para medirlas. Y esto es así porque sentirse de una clase u otra atiende a cuestiones más engorrosas que la mera observación de los bienes o recursos poseídos. Por ejemplo, la negativa a ubicarse en el estrato social más bajo (el de mayores necesidades) solo puede entenderse a la luz del estigma de la pobreza; la aparente vergüenza de reconocerse como parte de la élite económica ha de revisarse desde la dominación que oculta esta negativa.

4 En palabras de Ruth Zimmerling: "Las necesidades básicas simplemente son características empíricas universales de los hombres mientras estos sean lo que son... $\mathrm{N}$ es una necesidad básica para x si y solo si, bajo las circunstancias dadas en el sistema socio-cultural $\mathrm{S}$ en el que vive $\mathrm{x} y$ en vista de las características personales $\mathrm{P}$ de $\mathrm{x}$, la no satisfacción de $\mathrm{N}$ le impide a $\mathrm{x}$ la realización de algún fin no contingente - es decir, que no requiere justificación ulterior - y, con ello, la persecución de todo plan de vida" (Zimmerling ,1990, p. 51). 
El hecho de que una proporción considerable de la población afirme pertenecer a una clase media tiene que ver con una deficiente dimensión de los problemas sociales que nos aquejan. Como señala Hugo Seleme, los ciudadanos no somos conscientes de la magnitud de las desigualdades que nos separan. Utilizando el método de representación del economista Jan Pen, Seleme propone un ejercicio para visualizar el alcance de las desigualdades; este ejercicio consiste en correlacionar la medida de fortuna y la de estatura con los datos de pobreza y desigualdad. Así, a una determinada fortuna corresponde una determinada altura. Su conclusión es contundente: nuestros países están habitados, mayormente, por enanos. El objetivo de esta analogía consiste en poder representarnos mentalmente las brechas que nos alejan, cuya percepción está distorsionada. En nuestra autopercepción y en la percepción de otros no solo influye el estigma de ciertas ideas vinculadas al mérito, sino que se reproducen estructuras de poder. Nos negamos a denunciar la injusticia de las desigualdades porque desconfiamos de los que se encuentran en peor situación que nosotros. Los oprimidos se atacan entre sí, mientras que las élites que concentran la riqueza se mantienen al margen:

La verdadera brecha desigualitaria no es la que existe entre quien está por debajo de la línea de pobreza o justo por encima de ella, por un lado, y el habitante de la clase media que todos los meses percibe un buen ingreso que le ha permitido ser propietario de algunos bienes. La verdadera grieta provocada por la desigualdad se sitúa entre quienes poseen fortunas gigantescas y el resto de los ciudadanos, cuyas diferencias de riquezas e ingresos desde esta perspectiva son insignificantes (Seleme, 2020, p. 89).

Las desigualdades, por tanto, para poder ser apreciadas como problemáticas públicas requieren ser percibidas con la gravedad y el daño que envuelven. Estos problemas de percepción, sin embargo, no solo se dan en el propio sujeto, sino en la posible mirada de los demás. En este sentido, el trabajo de Dévora Imhoff, "Contribuciones de un abordaje psico-político a la comprensión de la dimensión subjetiva de la desigualdad social" (2020, pp. 49-64), subraya la importancia de considerar las experiencias subjetivas que se desarrollan en los escenarios de las desigualdades. Si bien 
es necesario contar con un análisis descriptivo de este fenómeno, esto no permite explicar cómo es que las dinámicas que resultan en estructuras de desigualdad se alojan en nuestras sociedades, cómo es que se normalizan y se justifican en el imaginario colectivo. Comprender cómo operan los sesgos cognitivos y las funciones adaptativas de la ceguera ante determinadas situaciones es fundamental si se pretende incidir de manera efectiva en las desigualdades. Por tanto, si bien el enfoque de necesidades básicas nos proporciona un eje para comprender e incidir en aquellos factores que fragmentan a la sociedad, parece ser un enfoque insuficiente para dar cuenta de las relaciones dinámicas de nuestras comunidades que favorecen la conservación y profundización de las desigualdades.

\section{La naturalización de las desigualdades estructurales}

Llegamos así a una de las cuestiones más destacadas en la obra objeto de esta reseña. El estudio diferenciado de las desigualdades permite dar cuenta de aquellas acciones que permitirían hacer balances y compensar las ventajas de unos y las desventajas de otros, pero, especialmente, permite poner de manifiesto la existencia de un sustrato que favorece a las desigualdades y que influirá en cualquier decisión estatal que se tome para mitigar sus efectos nocivos.

Probablemente nadie negaría que nuestras sociedades son profundamente desiguales. Nadie pondría en duda que hay ciertos sectores de la población que son marginados y que viven en condiciones indignas. Por tanto, si bien hay obstáculos en la autopercepción y en la percepción de las desigualdades que influyen en que se diluya su urgencia, hay otros obstáculos que no tienen que ver con la capacidad de ver, sino con el no querer ver: "En este caso, el hecho de no ver no es un problema de tipo físico, sino moral, político" (Lariguet, 2020, p. 20).

En consonancia con lo anterior, Imhoff propone adentrarse en los factores psico-sociales y psico-políticos latentes en la justificación y reproducción de las desigualdades. Hay una configuración de la estructura social que naturaliza las diferencias y las jerarquías entre las personas y que determina nuestra comprensión y evaluación de las desigualdades. 
Debido a la extensión y al impacto directo que estas tienen en la vida de las personas es razonable esperar que los más afectados sean quienes alcen la voz para desmontar prejuicios y para reclamar estrategias y acciones estatales para revertir esta situación. Sin embargo, en nuestras sociedades observamos la perseverancia de un discurso que justifica las desigualdades, que legitima las diferencias de trato, y que tiene una gran aceptación en las personas de los sectores sociales más vulneralizados. De ahí que Imhoff cuestione “ ¿No sería esperable que las personas beneficiadas por el orden desigual sean quienes lo defiendan y justifiquen, mientras que las personas en desventaja social lo critiquen y cuestionen?" (Imhoff, 2020, p. 55). Más allá del juicio rápido sobre lo absurdo de esta situación es interesante reparar en la función adaptativa que hay detrás de comportamientos y actitudes como estos. La autora señala que la legitimación de las desigualdades, su aceptación como algo natural e inevitable, reduce nuestros sentimientos de indignación moral y de culpa ante ellas. Esto explicaría por qué convivimos tan espontáneamente con situaciones que deberían dolernos, sernos intolerables. Sin embargo, como señala Amartya Sen "la adaptación de las percepciones y expectativas tiende a desempeñar un papel clave en la perpetuación de las desigualdades sociales” (2011, p. 313), así que es fundamental resistir a este señuelo.

La justificación de las desigualdades afecta la posibilidad de que la ciudadanía exija una mayor atención sobre estas, pero también afecta la propia perspectiva de las instituciones y del Derecho sobre este fenómeno. Llamosas pone de manifiesto cómo el progreso constitucional y codificador, por ejemplo, se consiguió a costa de ocultar las desigualdades, suponiendo un sujeto universal que formalmente es igual a los demás, pero que materialmente es excluyente. Las desigualdades han pasado a ser invisibles o invalidadas. Marisa Fassi y Angélica Peñas presentan un ejemplo claro de dicha invalidación. La pandemia ha mostrado el calado de las desigualdades; sus efectos han recrudecido las necesidades no satisfechas de determinados colectivos, así como su marginalidad en el espacio público. Muchas personas que realizan trabajo sexual en la calle fueron excluidas de las ayudas de emergencia pese a encontrarse en situación de extrema vulnerabilidad. En algunos países se decidió descartar el trabajo sexual como una actividad que cabe en la categoría de trabajo. Detrás de esta decisión que excluye hay 
una historia de desigualdades estructurales que criminaliza la prostitución y deshumaniza a quienes la ejercen. Hay así una idea hegemónica acerca de lo que cuenta como actividades generadoras de ingresos que determina las políticas públicas y las decisiones institucionales, además del imaginario social del trabajo y la vida digna (Fassi y Peñas, 2020, p. 115). La esfera pública se muestra así como un espacio de representación y desarrollo de desigualdades.

Por supuesto, esto no pretende ser una invitación al pesimismo y a dar por hecho que estas estructuras de poder que oprimen a algunos y que priorizan los intereses de otros existen y existirán de manera indefinida en nuestras comunidades. Al inicio destacaba cómo el libro pretende ser una invitación a la acción y al cambio, no al inmovilismo. Lo que sucede es que la transformación social exige develar los complejos mecanismos que fomentan las desigualdades y mostrar las dimensiones del reto que tenemos delante. Para ello se requiere la confluencia de múltiples cuestiones, pero una fundamental tiene que ver con la formación y la sensibilización de quienes están a cargo de la toma de decisiones.

\section{De la reflexión a la acción para la transformación social. ¿Qué educación para el agente práctico?}

En este sentido, Silvana Begala y Mariana Manzo, en "Acceso a la justicia de las personas migrantes: una práctica de extensión-comunicación" (2020, pp. 123-139), presentan un trabajo que cuestiona la capacidad de la educación jurídica tradicional para poder estar a la altura de las exigencias de nuestro tiempo. Un modelo educativo y un currículo que se encuentren dirigidos de manera exclusiva al conocimiento del Derecho positivo difícilmente permitirá a los futuros juristas ser conscientes de las desigualdades y del papel decisivo que juega en Derecho, ya sea como instrumento transformador o como uno que mantiene esas desigualdades. De ahí que insistan en la oportunidad de formar perfiles de profesionales del Derecho que sean reflexivos y comprometidos socialmente, que posean habilidades cognitivo-críticas y habilidades que les permitan valorar las repercusiones de la aplicación del Derecho (Begala y Manzo, 2020, p. 131). Para ello, sin 
embargo, se requiere romper con ese modelo que recompensa la indiferencia y la falta de empatía. Es necesario que el Derecho se meta de lleno en el terreno de las desigualdades, soltando la añeja aspiración — por otra parte, puramente ideológica o enmascaradora- de construir una disciplina aislada, formal y neutral.

\section{Conclusiones}

La obra que se ha reseñado en estas páginas es valiosa en tanto que nos permite repensar la fiabilidad de nuestras creencias y percepciones, revisar nuestros sesgos y adaptaciones, así como cuestionar los discursos que presentan las desigualdades como algo inherente al ser humano y a la sociedad. Además, nos permite identificar las herramientas teórico/prácticas que requerimos para acercarnos a este complejo fenómeno, así como las limitaciones que corresponde afrontar. Al tiempo, las autoras y autores de la obra enuncian las dificultades y los retos que tenemos por delante para, desde la academia, poder contribuir a la acción frente a las desigualdades. El camino que queda por recorrer es todavía muy largo, pero aportaciones como las que he intentado mostrar aquí aportan luz sobre el sendero que debemos tomar y los pasos que lo deben recorrer.

\section{Bibliografía}

Aristóteles (1998). Ética Nicomáquea. Madrid: Editorial Gredos.

Assusa, G. (2020). El ser social y la conciencia. Preguntas sobre la dimensión simbólica de la desigualdad social. En E. Llamosas y G. Lariguet (eds.), Problemas en torno a la desigualdad. Un enfoque poliédrico (pp. 33-47). Córdoba: Universidad Nacional de Córdoba.

Begala, S. y Manzo, M. (2020). Acceso a la justicia y de las personas migrantes: una práctica de extensión-comunicación. En E. Llamosas y G. Lariguet (eds.), Problemas en torno a la desigualdad. Un enfoque poliédrico (pp. 123-139). Córdoba: Universidad Nacional de Córdoba. 
Fatauros, C. (2020). Las desigualdades que nos duelen, ¿son acaso desigualdades que deberíamos tolerar? En E. Llamosas y G. Lariguet (eds.), Problemas en torno a la desigualdad. Un enfoque poliédrico (pp. 93-106). Córdoba: Universidad Nacional de Córdoba.

Fassi, M. y Peñas, A. (2020). Colonialidad y pandemia 2.0. Retos para repensar los relatos sobre el trabajo sexual en Argentina. En E. Llamosas y G. Lariguet (eds.), Problemas en torno a la desigualdad. Un enfoque poliédrico (pp. 109-121). Córdoba: Universidad Nacional de Córdoba.

Friedman, M. y Friedman, R. (1980). Free to Choose. A Personal Statement. Nueva York: Harcourt Brace Jovanovich.

Imhoff, D. (2020). Contribuciones de un abordaje psico-político a la comprensión de la dimensión subjetiva de la desigualdad social. En E. Llamosas y G. Lariguet (eds.), Problemas en torno a la desigualdad. Un enfoque poliédrico (pp. 49-64). Córdoba: Universidad Nacional de Córdoba.

Lariguet, G. (2018). Sócrates, tipologías de intelectuales y política: algunas referencias al caso argentino. Literatura: teoría, historia, crítica, 20(2), 185-220.

Lariguet, G. (2020). Prólogo. La desigualdad: una triste e infinita melodía. En E. Llamosas y G. Lariguet (eds.), Problemas en torno a la desigualdad. Un enfoque poliédrico. Córdoba: Universidad Nacional de Córdoba, 13-29.

Llamosas, E. (2020). Las desigualdades jurídicas: de naturales a invisibles, entre el Antiguo Régimen y la codificación. En E. Llamosas y G. Lariguet (eds.), Problemas en torno a la desigualdad. Un enfoque poliédrico (pp. 65-78). Córdoba: Universidad Nacional de Córdoba.

Llamosas, E. y Lariguet, G. (2020). Problemas en torno a la desigualdad. Un enfoque poliédrico. Córdoba: Universidad Nacional de Córdoba.

Nino, C. (1990). Autonomía y necesidades básicas. Doxa: Revista de filosofía del Derecho, 7, 21-34.

Rawls, J. (1999). A Theory of Justice. Massachusetts: The Belknap Press of Harvard University Press.

Sandel, M. (2020). La tiranía del mérito. ¿Qué ha sido del bien común? Barcelona: Editorial Debate. 
Seleme, H. (2020). El lugar de la democracia y la magnitud de la desigualdad. En E. Llamosas y G. Lariguet (eds.), Problemas en torno a la desigualdad. Un enfoque poliédrico (pp. 79-92). Córdoba: Universidad Nacional de Córdoba.

Sen, A. (2011). La idea de justicia. Buenos Aires: Editorial Taurus.

Wiggins, D. y Dermen, S. (1987). Needs, need, needing. Journal of Medical Ethics, 13, 62-68.

Zimmerlink, R. (1990). Necesidades básicas y relativismo moral. Doxa: Revista de filosofía del Derecho, 7, 35-54. 